\title{
Erk1/2 activation and modulation of STAT3 signaling in oral cancer
}

\author{
IOANNIS GKOUVERIS ${ }^{1}$, NIKOLAOS NIKITAKIS ${ }^{1}$, MARIA KARANIKOU ${ }^{2}$, \\ GEORGE RASSIDAKIS $^{2}$ and ALEXANDRA SKLAVOUNOU ${ }^{1}$
}

\author{
${ }^{1}$ Department of Oral Pathology and Surgery, Dental School; ${ }^{2}$ First Department of Pathology, \\ Medical School, National and Kapodistrian University of Athens, Athens 11527, Greece
}

Received April 6, 2014; Accepted July 16, 2014

DOI: $10.3892 /$ or.2014.3440

\begin{abstract}
Constitutive activation of the signal transducer and activator of transcription 3 (STAT3) signaling pathway possesses confirmed oncogenic potential in oral squamous cell carcinoma (OSCC). Crosstalk with other molecular pathways contributes to STAT3 regulation in cancer. The effects of mitogen-activated protein kinases (MAPKs) and particularly extracellular signal-regulated kinase 1/2 (Erk1/2) on STAT3 signaling in OSCC have not been thoroughly investigated. The present study examined the effects of Erk1/2 modulation on STAT3 signaling and cell growth in OSCC cells. Constitutive expression levels of phosphorylated (tyrosine and serine) and total STAT3, Erk1/2 and cyclin D1 were assessed in OSCC cell lines. Erk1/2 modulation was achieved by pharmacological agents; siRNA silencing against Erk1/2 was also performed. Cell proliferation and viability were assessed. Erk1/2 inhibition with either U0126 treatment or specific siRNA silencing resulted in decreases in p-ser STAT3 and cyclin D1 levels and increases in p-tyr STAT3 in OSCC cells. Moreover, Erk1/2 inhibition resulted in a dose-dependent reduction in OSCC cell growth and viability. Erk1/2 induction had the opposite effects. Taken together, these results are supportive of an active crosstalk between the oncogenic Erk1/2 and STAT3 pathways in OSCC, the significance of which requires further investigation.
\end{abstract}

\section{Introduction}

Oral cancer is the sixth most common cancer in the world and the incidence of new cases indicates a continuing rise in developing countries (1). Several genetic and epigenetic alterations

Correspondence to: Dr Ioannis Gkouveris, Department of Oral Pathology and Surgery, Dental School, National and Kapodistrian University of Athens, 2 Thivon Street, Goudi, Athens 11527, Greece E-mail: igkouver@gmail.com

Key words: extracellular signal-regulated kinase 1/2, signal transducer and activator of transcription 3, crosstalk, cell signaling, cell proliferation, oral cancer underlie the progressive acquisition of a malignant phenotype in head and neck squamous cell carcinoma (HNSCC) (2). The molecular dissection of aberrant signaling networks, including EGFR, Ras, NF- $\kappa \mathrm{B}, \mathrm{Wnt} / \beta$-catenin, TGF- $\beta$, and PI3K-AKT-mTOR signaling pathways, has increased our understanding of the basic mechanisms controlling HNSCC progression $(2,3)$.

Signal transducer and activator of transcription (STAT) proteins constitute a family of transcription factors, which exist in the cell as latent cytoplasmic transcription factors and become activated in response to stimulation by cytokines and growth factors $(4,5)$. Once bound to their receptors, STATs become phosphorylated, they dissociate from the receptor and form homo- or heterodimers (4-7). STAT dimers then translocate to the nucleus, where they interact with the promoters of target genes, thus regulating transcription (4-7).

Phosphorylation of STAT molecules on a tyrosine residue is the first critical event for their activation and has been convincingly shown to correlate with STAT DNA binding and transcriptional activity (4-7). In contrast, STAT serine phosphorylation, which may also occur in response to growth factor and cytokine stimulation, has been associated mainly with negative regulation of STAT activity (8-11). Other authors have indicated that STAT1 and STAT3 are often phosphorylated in serine residues, resulting in further STAT activation (12).

STAT signaling has been found to be involved in oncogenesis $(13,14)$. STAT3 persistent activation has been convincingly shown to contribute to a malignant phenotype (13). Constitutive activation of STAT3 has been associated with tumorigenesis in various types of human cancer by stimulating specific target genes that enhance cell proliferation and prevent apoptosis (13-15).

There is compelling evidence that STAT3 constitutive activation, mainly associated with aberrant TGF- $\alpha /$ EGFR signaling, is linked to HNSCC development and growth (2,14-18). Previous findings suggested the existence of EGFRindependent STAT oncogenic properties in HNSCC, including autocrine/paracrine cytokine (IL-6, IL-10 or IL-22) stimulation or signaling through 7 nicotinic and erythropoietin receptor pathways (17,19-23). Cytokine receptors are constitutively associated with members of the Janus kinase (JAK) family of protein tyrosine kinases, whereas growth factor receptors have intrinsic tyrosine kinase activity (5). 
Mitogen-activated protein kinase (MAPK) pathways are evolutionarily conserved kinase modules that link extracellular signals to the machinery that controls fundamental cellular processes such as growth, proliferation, differentiation, migration and apoptosis (24). MAPKs phosphorylate serine and threonine residues of specific target proteins (25). MAPKs are classified into three major subfamilies, including extracellular signal-regulated kinases (ERKs), p38 MAPKs, and c-Jun $\mathrm{NH}_{2}$-terminal kinases (JNKs) (25-27).

Previous studies supported an association between activation of specific members of the MAPK family and negative regulation of STAT3 signaling in various cell types $(8,28-34)$. Chung et al showed that ERKs phosphorylate STAT3 on serine 727 (Ser727) in vitro and in vivo, while inhibiting STAT3 tyrosine phosphorylation (8). Similarly, Jain et al confirmed that ERKs induce STAT3 serine phosphorylation and suppress STAT3 tyrosine phosphorylation, DNA binding and transcriptional activity induced by Src or Jak-2 (28). Moreover, Sengupta et al provided evidence that activated ERKs induce a rapid downregulation of IL-6-mediated STAT3 signaling through inhibition of the upstream JAK kinases in several cell lines (29). Recently, Gough et al found that the MEK-ERK pathway is required for activated Ras-induced phosphorylation of mitochondrial STAT3 on Ser727, an important process during cellular transformation (35). Also, Xue et al suggested that treatment of human lung adenocarcinoma cells with RY10-4 affected Bcl-2 family members, caspases, MMPs, TIMPs expression and ROS production by inhibiting STAT3 activities through ERK and p38 pathways (36). Finally, Lee et al found that blockade of MEK1/2-Erk1/2-RSK2 signaling by silybin resulted in a reduced activation of $\mathrm{NF}-\kappa \mathrm{B}$, activator protein-1, and STAT3 in melanoma cells (37). Collectively, there is compelling evidence to suggest that MAPK, especially ERK, activation has an inhibitory effect on STAT3 signaling, manifested by downregulation of STAT3 tyrosine phosphorylation and induction of STAT3 serine phosphorylation, in various cell types including cancer cells.

The aim of the present investigation was to evaluate whether oncogenic constitutive STAT3 signaling in oral squamous cell carcinoma (OSCC) cells can be modulated by regulation of specific MAPKs. The expression and activation status of STAT3 and Erk1/2 in HNSCC cell lines were recorded and the effects of selective Erk1/2 inhibition or activation on STAT3 signaling and cellular proliferation were monitored, in an effort to elucidate important molecular aspects of oral cancer with potential therapeutic implications.

\section{Materials and methods}

Cell lines and cell culture. Experiments were performed using established cell lines of human HNSCC (SCC9 and SCC25) obtained from the American Type Culture Collection (ATCC; Manassas, VA, USA). Cells were cultured in a 1:1 mixture of Ham's F12 and DMEM containing 10\% fetal bovine serum (FBS), 100 units of penicillin, and $400 \mathrm{ng} / \mathrm{ml}$ hydrocortisone (Sigma Chemical Co., St. Louis, MO, USA) at $37^{\circ} \mathrm{C}$ in a $5 \% \mathrm{CO}_{2}$ air atmosphere. Cells were subcultured by disaggregation with trypsin $(0.1 \%)$-EDTA $(0.01 \%)$ in phosphate-buffered saline (PBS) at $\mathrm{pH} 7.5$.
Selective inhibition of Erk1/2. Cells were plated in 6-well plates at a density of $5 \times 10^{4}$ cells/well and were allowed to grow to $80 \%$ confluency. Then, they were treated either with the vehicle alone (DMSO at a maximum concentration of $0.1 \%$ ) or with the selective MAPK (Erk1/2) inhibitor U0126 (Calbiochem, San Diego, CA, USA) at concentrations of 20 and $50 \mu \mathrm{M}$ for $24 \mathrm{~h}$.

Selective induction of Erk1/2 MAPK. Cells were plated in 6 -well plates at a density of $2 \times 10^{5}$ cells/well and were allowed to grow to $80 \%$ confluency. They were then treated either with the vehicle alone (DMSO at a maximum concentration of $0.1 \%$ ) or with the selective MAPK (Mek1/2) inducer (ProSpec, Israel) at concentrations of 2 and $5 \mu \mathrm{M}$ for $48 \mathrm{~h}$.

siRNA transfection. The human Erk1 and Erk2 specific siRNAs were based on NCBI Reference Sequences (GenBank: Erk1: NM_002746 and Erk2: NM_002745). Erk1/2 siRNA and scrambled control siRNA (siControl) were purchased from Qiagen. All siRNA transfections were performed using Lipofectamine 2000 (Invitrogen), according to the manufacturer's protocol, with final siRNA concentrations of 1 and $2.5 \mu \mathrm{M}$. OSCC cells were grown to mid-log phase and were transiently transfected $\left(2 \times 10^{6}\right.$ cells $)$ with $50 \mu \mathrm{g}$ of the empty vector or siRNA using Nucleofector reagents (Amaxa Biosystems, Gaithersburg, MD, USA). Cells were collected at $48 \mathrm{~h}$ and whole lysates were analyzed by western blotting.

Western blot experiments. Cells were washed twice with ice-cold PBS, followed by lysis with radioimmunoprecipitation assay buffer (50 mM Tris $\mathrm{pH} 7.4,150 \mathrm{mM} \mathrm{NaCl}$, $1 \%$ Triton $\mathrm{X}-100,1 \%$ deoxycholic acid, sodium salt, $0.1 \%$ sodium dodecyl sulfate, $100 \mathrm{mg} / \mathrm{ml}$ phenylmethylsulfonyl fluoride, $1 \mathrm{mg} / \mathrm{ml}$ aprotinin, $1 \mathrm{mM}$ dichlorodiphenyltrichloroethane and $1 \mathrm{mM}$ sodium orthovanadate) for $10 \mathrm{~min}$ at $4^{\circ} \mathrm{C}$. The wells were scraped, and recovered cell products were centrifuged at $40,000 \mathrm{x}$ g for $15 \mathrm{~min}$ at $4^{\circ} \mathrm{C}$. Recovered proteins were measured and equalized using Bio-Rad Protein Assay (Bio-Rad Laboratories, Richmond, CA, USA) as per the manufacturer's instructions.

Western blotting was performed using antibodies against: total STAT3, phospho-STAT3 (Tyr705), phospho-STAT3 (Ser727), total p44/42(Erk1/2) (Cell Signaling, Beverly, MA, USA), phospho-Erk1/2 MAPK, (Upstate, Charlottesville, VA, USA), cyclin D1 (Cell Signaling) and $\beta$-actin (Sigma Chemical).

Cell proliferation and viability. Cells were counted with a hemocytometer under inverted microscope. Cell viability after treatment was determined by the trypan blue dye exclusion test $24 \mathrm{~h}$ after each treatment. All assays were performed in quadruplicate and results are reported as the mean \pm SD.

\section{Results}

Effects of U0126 inhibitor on Erk1/2, STAT3 and cyclin D1 protein expression and activation. The expression and activation status of Erk1/2 in OSCC cells was examined first. According to western blotting experiments, Erk1/2 (p42/44) total and phosphorylated (activated) protein levels were 


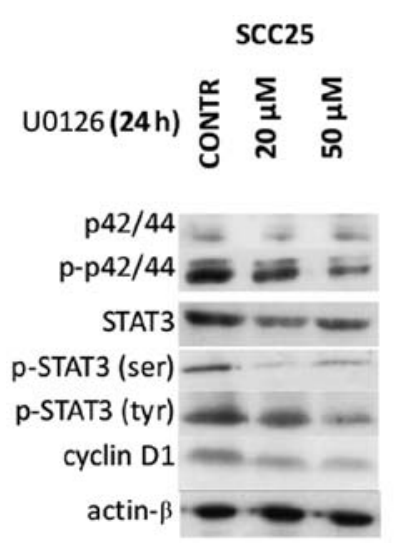

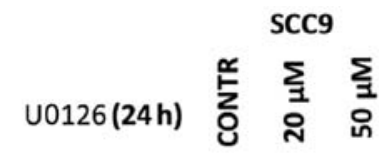

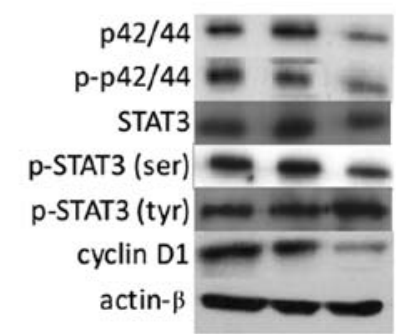

Figure 1. Effect of U0126 inhibitor on STAT3 phosphorylation and cellular activity. Treatment of cells with U0126 for $24 \mathrm{~h}$ had a slight decreasing effect on total Erk1/2(p42/44) levels in SCC25, while, as expected, it led to reduced levels of phospho-Erk1/2 more pronounced at the highest concentration. Selective inhibition of ERK also resulted in downregulation of phospho-STAT3 (Ser727). Inhibition of ERK did not affect the constitutive levels of phospho-STAT3 (Y705) in SCC25. In contrast, in the SCC9 cell line, STAT3 phosphorylation in Tyr705 presented moderate increase. Cyclin D1 levels decreased in both cell lines in a dose-dependent manner.

detected in both OSCC cell lines tested (SCC9 and SCC25). Total, tyrosine phosphorylated (p-tyr) and serine phosphorylated (p-ser) STAT3, as well cyclin D1 protein levels were also observed in both cell lines (Fig. 1).

The effectiveness of Erk1/2 inhibition was then assessed. Treatment of oral SCC25 cells with the Erk1/2 inhibitor U0126 for $24 \mathrm{~h}$ resulted in inhibition of Erk1/2 phosphorylation, which was more pronounced at the highest concentration used $(50 \mu \mathrm{M})$. A decrease in total Erk1/2 protein expression levels following 20 or $50 \mu \mathrm{M}$ of U0126 treatment was also noted in SCC25 cells. On the other hand, U0126 treatment of oral SCC 9 cells caused less notable effects on Erk1/2 protein expression and phosphorylation with decreases observed only at the highest concentration $(50 \mu \mathrm{M})$ (Fig. 1).

The effectiveness of Erk1/2 inhibition on STAT3 protein expression and activation levels was also examined. In oral SCC25 cells, a significant reduction of p-ser STAT3 was detected after $24 \mathrm{~h}$ of treatment with 20 or $50 \mu \mathrm{M}$ of U0126; in contrast, p-tyr STAT3 levels were not significantly affected. On the other hand, treatment of SCC9 cells with U0126 led to a decrease of p-ser STAT3 only when a concentration of $50 \mu \mathrm{M}$ was used, along with a moderate increase in p-tyr STAT3 levels. Total STAT3 levels were not affected by U0126 treatment in either cell line (Fig. 1).

Moreover, western blot analysis demonstrated that inhibition of Erk1/2 was associated with decreased levels of cyclin D1 expression in a dose-dependent manner in both cell lines. In contrast, the levels of actin remained stable throughout the treatment, indicating that the observed effects on the aforementioned proteins were not caused by a nonspecific reduction of protein expression (Fig. 1).

Hence, Erk1/2 inhibition by U0126 treatment was more potent in oral SCC25 cells and was associated with a decrease in p-ser STAT3 and cyclin D1 levels without affecting p-tyr STAT3 levels. In contrast, U0126 treatment of oral SCC9 cells appeared to be less effective in reducing Erk1/2 phosphorylation;

\section{Viability}

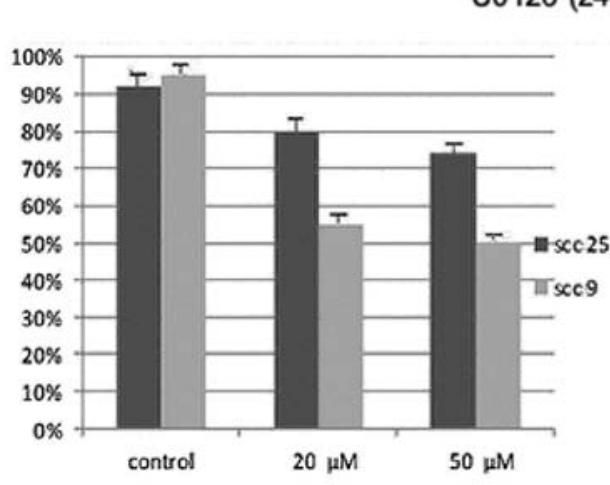

Cell number

U0126 (24 h)

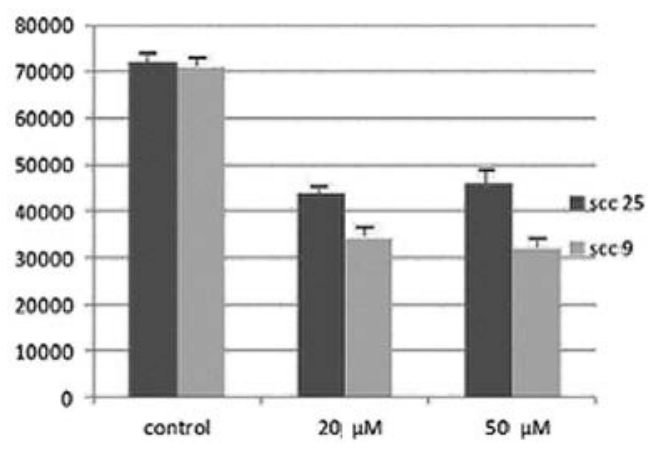

Figure 2. Effect of U0126 on cell growth and viability. Treatment of cells with Erk1/2 inhibitor U0126 affected both cell viability and absolute number of living cells. The reduction appeared to be more prominent in the SCC9 cell line. Columns, average of four determinations; bars, SD.

nonetheless, it induced decreases in p-ser STAT3 levels and cyclin D1 protein expression as well as increases in p-tyr STAT3 levels at the highest concentration used.

Effects of U0126 inhibitor on cell growth and viability. Treatment with U0126 for $24 \mathrm{~h}$ resulted in a statistically significant $(\mathrm{P}<0.05)$ dose-dependent reduction in cell growth (absolute number of cells) and cell viability (number of viable cells) in both cell lines tested. The reduction appears to be more prominent in the oral SCC9 cell line (Fig. 2).

Effects of Erk1/2 siRNA silencing on STAT3 and cyclin D1 protein expression and activation. In order to corroborate the results from the pharmacological inhibition of Erk1/2, specific inhibition was performed by siRNA-targeting of Erk $1 / 2$ in both cell lines. Following $48 \mathrm{~h}$ of transfection, western blotting revealed that si-RNA against Erk1/2 efficiently silenced Erk1/2 causing dose-dependent decreases in total and phosphorylated p42/44 Erk1/2 protein levels compared to control-transfected cells in both cell lines (Fig. 3).

Decreases in Erk1/2 protein expression and phosphorylation correlated with a decrease in p-ser STAT3 in both cell lines, after $48 \mathrm{~h}$ of treatment with $2.5 \mu \mathrm{M}$ of specific siRNA against Erk1/2. Regarding STAT3 tyrosine phosphorylation, an upregulation of p-tyr-STAT3 protein levels was detected, particularly at the highest concentration, in oral SCC9 cells. On the other hand, siRNA treatment against Erk1/2 did not appear to cause any observable change in p-tyr-STAT3 levels 


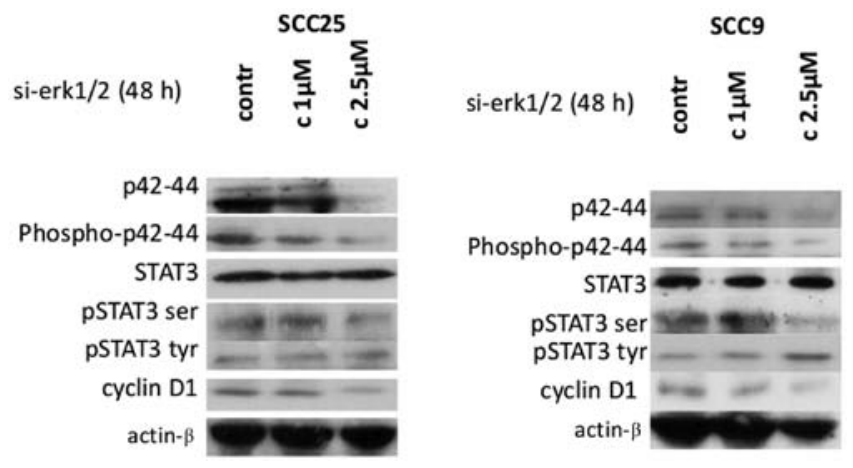

Figure 3. Effect of Erk1/2 silencing (siRNA) on STAT3 phosphorylation and cellular activity. After siRNA transfection $(48 \mathrm{~h})$, western blotting revealed that the siErk1/2 inhibited total protein expression compared with control transfection experiments. Moreover, the level of phospho-Erk1/2 was markedly reduced in these cells in a dose-dependent manner. The decrease in Erk1/2 expression/phosphorylation correlated with a decrease of phospho-STAT3 (Ser727) and cyclin D1 expression while p-tyr-STAT3 protein levels were detected, especially at the highest concentration, in oral SCC9 cells.

in oral SCC25 cells. Total STAT3 protein levels were not affected in either cell line (Fig. 3).

Furthermore, western blot analysis demonstrated that silencing of Erk1/2 was associated with substantially decreased levels of cyclin D1 protein expression in a dose-dependent manner in both cell lines. Finally, the levels of actin remained stable (Fig. 3). Therefore, specific silencing of Erk1/2 resulted in a decrease in p-ser STAT3 and cyclin D1 levels in both cell lines and an increase in p-tyr-STAT3, particularly in oral SCC9 cells.

Effects of silencing Erk1/2 on cell growth and viability. Similar to the effects of chemical inhibition with U0126, $48 \mathrm{~h}$ of treatment with siRNA against Erk1/2 resulted in a dose-dependent reduction in cell growth and cell viability in both cell lines $(\mathrm{P}<0.05)$ (Fig. 4).

Effects of Erk1/2 induction on STAT3 and cyclin D1 protein expression and activation. In order to further investigate the significance of Erk1/2 for STAT3 and cyclin D1 modulation, pharmacological induction of Erk1/2 using active MEK1/2 was performed. Treatment of cells with selective MEK1/2 inducer efficiently upregulated phosphorylated Erk1/2 levels in a dose-dependent manner in both cell lines without affecting total Erk1/2 levels, as expected (Fig. 5).

Treatment of both cell lines with Erk1/2 inducer for $48 \mathrm{~h}$ resulted in significant induction of STAT3 phosphorylation on Ser727, especially at a concentration of $5 \mu \mathrm{m} / \mathrm{ml}$. In contrast, p-tyr-STAT3 levels appeared to decrease after treatment with active MEK1/2. Total STAT3 levels were not affected by Erk1/2 induction in either cell line (Fig. 5).

With regards to cyclin D1, active MEK1/2 treatment of both cell lines for $48 \mathrm{~h}$ caused an upregulation in cyclin D1 expression levels, particularly at the higher concentration. Finally, actin protein levels remained stable throughout treatment (Fig. 5). Thus, Erk1/2 induction caused upregulation of p-ser STAT3 and cyclin D1 levels in both cell lines and a decrease in p-tyr-STAT3.
Viability

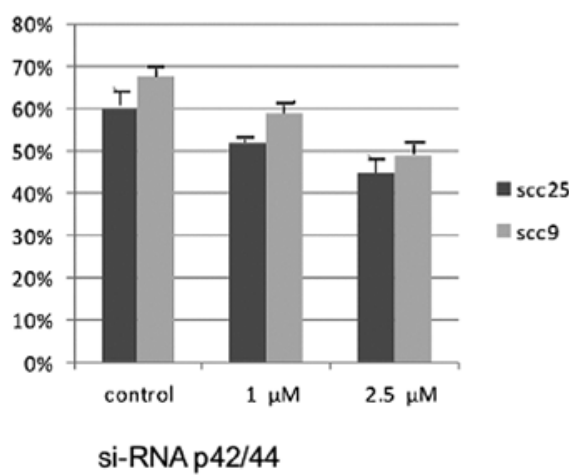

Cell number

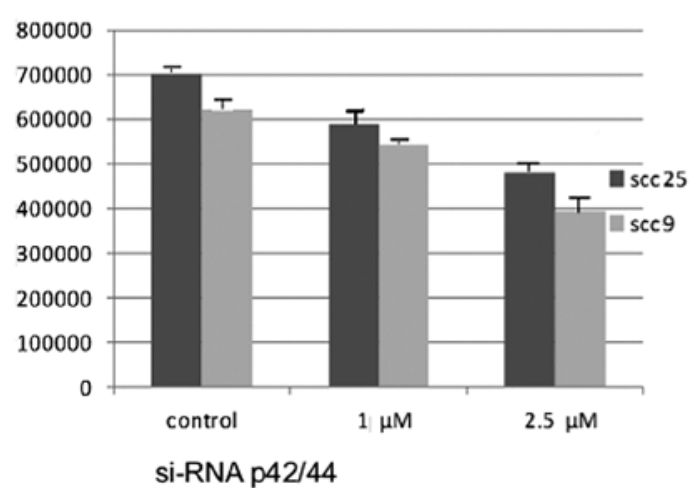

Figure 4. Effect of silencing Erk1/2 on cell growth and viability. Treatment of cells with siErk $1 / 2$ decreased the absolute number of living cells and reduced the percentage of cell viability levels in both cell lines. Columns, average of four determinations; bars, SD.

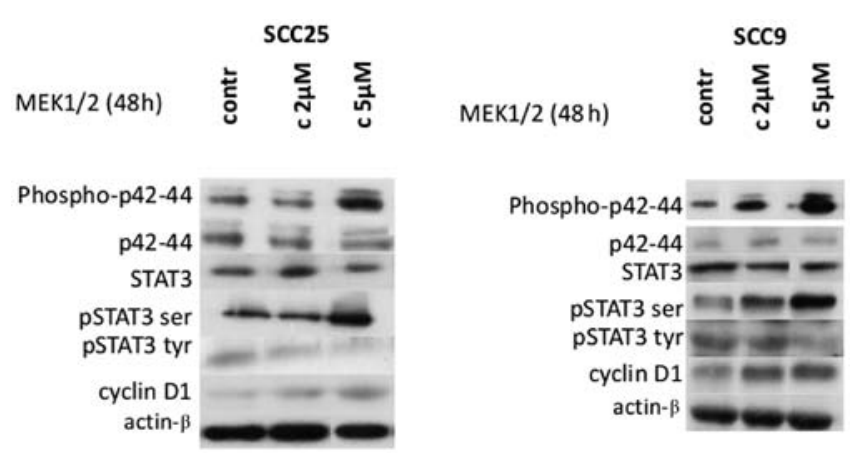

Figure 5. Effect of Erk1/2 induction on STAT3 phosphorylation and cellular activity. Induction of Erk1/2 with active MEK1/2 for $48 \mathrm{~h}$ successfully enhanced phospho-Erk1/2. Furthermore, induction of ERK resulted in upregulation of phospho-STAT3 (Ser727) and in slight downregulation of STAT3 tyrosine phosphorylation. Treatment of both cell lines for $48 \mathrm{~h}$ also caused a slight upregulation in cyclin D1 expression levels.

Effects of Erk1/2 induction on cell growth and viability. Active MEK1/2 treatment at the highest concentration for $48 \mathrm{~h}$ resulted in a significant dose-dependent increase in cell growth, which was more prominent in the oral SCC25 cell line $(\mathrm{P}<0.05)$. On the contrary, treatment of cells with active MEK $1 / 2$ for $48 \mathrm{~h}$ did not appear to induce notable changes in cell viability in either cell line (Fig. 6). 


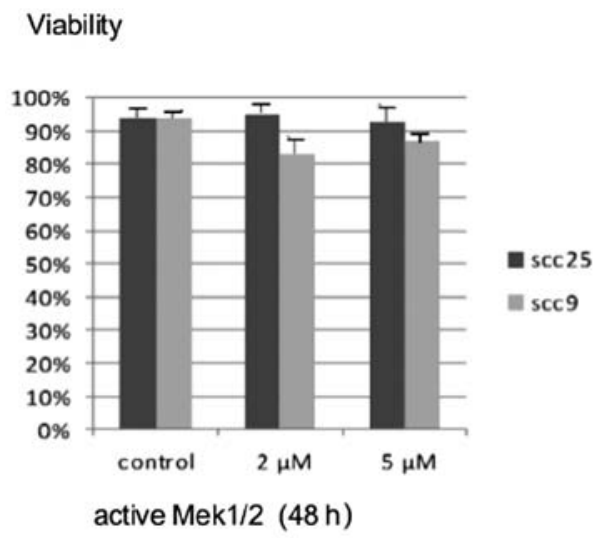

\section{Cell number}

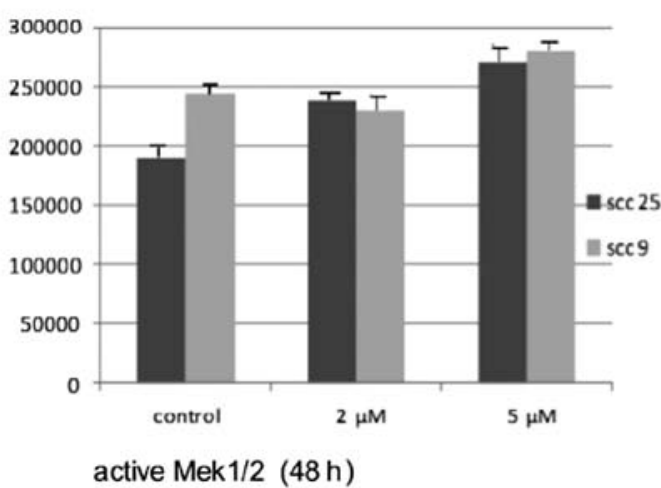

Figure 6. Effect of Erk1/2 induction on cell growth and viability. Induction of Erk1/2 with selective MEK1/2 inducer affected absolute number of living cells and led to cell growth, particularly at the higher concentration in both cell lines. Treatment of cells with active MEK1/2 for $48 \mathrm{~h}$ did not appear to cause any change in cell viability in either cell line. Columns, average of four determinations; bars, $\mathrm{SD}$

\section{Discussion}

The oncogenic role of MAPK signaling pathway has been the focus of numerous studies indicating that aberrant MAPK expression alters differentiation and deregulates proliferation and apoptosis in several types of cancer $(38,39)$. In particular, ERK is a member of an oncogenic pathway activated by the upstream oncoproteins Ras and Raf $(24,40)$. Upon activation, Erk1/2 phosphorylates either cytoplasmic downstream targets, including p90 ribosomal S6 kinase, or nuclear substrates $(24,41)$. In the nucleus, ERK phosphorylates an array of targets, including transcription factors and the family of mitogen and stress-activated protein kinases (MSKs) (42). ERK nuclear targets also include the Ternary Complex Factor (TCF), which plays a crucial role in enhancing expression of the immediate early genes, such as c-Fos and c-Myc $(39,43)$.

The significance of the MEK/ERK pathway has been shown in various types of cancer, such as liver, prostate and malignant melanoma cancer cell growth in vitro and in vivo (44-46). For example, knockdown of serine/threonine kinase Mirk/Dyrk1B by siRNA in either ovarian cancer or non-small cell lung cancer (NSCLC) cells led to upregulated activation of c-Raf-MEK-ERK, followed by increased growth rate (47). Induction of anti-apoptotic proteins, such as Bcl2, Bclxl and inhibition of pro-apoptotic factors, including Bad, has been correlated with the oncogenic potential of ERK activity (48).

Deregulation of ERK has also been described in HNSCC and various studies have suggested that ERK inhibition correlates with reduced proliferation and induced apoptosis in OSCC $(49,50)$. Wang et al suggested that overexpression of activated Erk1/2 and cyclin D1 might be related to cell cycle regulation and cell proliferation in OSCC (51). Bancroft et al (52) showed the involvement of Erk $1 / 2$ in AP-1 and NF- $\mathrm{KB}$ induction of VEGF expression in OSCC cell lines SCC9 and SCC11, and Duvvuri et al (53) found that overexpression of Erk1/2, induced by receptor-activated calcium-dependent chloride channel (TMEM16A), is associated with enhanced cell proliferation in HNSCC. Recently, Li et al demonstrated that knockdown of kinase suppressor of Ras 1 and suppression of the Raf-MEK-ERK pathway reduced proliferation and induced apoptosis in OSCC cells (54).

In agreement with the aforementioned findings, our results are in support of the oncogenic role of ERK in oral cancer. In the present study, possible effects of ERK modulation on OSCC cell proliferation and viability were assessed. In particular, Erk1/2 inhibition caused a dose-dependent decrease in the absolute number of living cells along with downregulation of cyclin D1 levels in both OSCC cell lines tested. These findings corroborate previous studies that demonstrated negative regulation of cell growth and cyclin D1 levels following Erk1/2 inhibition by means of U0126 treatment $(44,47,55,56)$.

In our study, selective siRNA Erk1/2 inhibition induced similar results to those of Erk1/2 pharmacological inhibition in a dose-dependent manner in both cell lines, followed by a corresponding decrease in the absolute number of living cells and cell viability levels. Using similar siRNA techniques, Bessard et al underlined the crucial role of the MEK/ERK pathway in liver cancer cell growth in vitro and in vivo demonstrating that RNAi-mediated Erk2 knockdown inhibits tumor cell growth (44). Similarly, Si et al showed that downregulation of Erk1/2 by siRNA inhibited the growth and invasion of human osteosarcoma cells, and found that the knockdown of Erk1/2 made cancer cells more sensitive to cisplatin treatment (57). Moreover, Dumesic et al reported that combined siRNA-induced knockdown of Erk1/2 caused epidermal hypoplasia and hypoproliferation without disrupting differentiation in human epidermis (58). Finally, Duvvuri et al found that genetic inactivation of Erk1/2 using siRNA abrogated the Erk1/2 mediated growth effects of TMEM16A in HNSCC (53).

Furthermore, we demonstrated that pharmacological induction of Erk1/2 appears to have the opposite effects. Notably, the increase in absolute cell number is accompanied by a relative increase in cyclin D1. In agreement, Gao et al suggested that activation of the c-Raf-MEK-Erk $1 / 2$ pathway leads to subsequent increase in cell cycle proteins (cyclin D1, p27kip1), accompanied by an increased growth rate and transition of cells from G0/G1 into the S phase of the cell cycle in both ovarian and non-small cell lung cancer (47). Similarly, Wang et al indicated that overexpression of activated Erk1/2 
and cyclin D1 proteins are involved in oral tongue carcinogenesis (51). Finally, Judd et al (59) reported that MEK1 activation enhances CD44 expression and promotes aggressiveness in HNSCC, while Katada et al (60) and Zuo et al (61) suggested that Erk1/2 activation correlates with increased cell migration and invasion in HNSCC.

A number of findings indicate that STAT3 is constitutively activated and participates in the regulation of cell proliferation, differentiation and apoptosis in $\operatorname{HNSCC}(17,18)$. Aberrant STAT3 activation, manifested by increases in STAT3 tyrosine phosphorylation, is considered as a potent promoter of HNSCC initiation and progression, thereby inhibition of STAT3 has been identified as a potential therapeutic target for the treatment of HNSCC $(16,18,62)$. STAT3 constitutive activation in HNSCC is driven by a number of upstream signal transduction pathways. Ligand-induced stimulation of the receptor results in phosphorylation of tyrosine residues within the receptor, which is the first critical event for their activation and has been shown to correlate with STAT DNA binding and transcriptional activity (4,5,7-9). The oncogenic potential of STAT3 depends mainly on the phosphorylation status of Tyr705 whereas the role of STAT3 serine phosphorylation is more controversial (7). There are several lines of evidence supporting a negative impact of Ser727 residue phosphorylation on STAT3 activity. Previous studies have demonstrated that Ser727 phosphorylation negatively regulates STAT3 tyrosine phosphorylation, which is required for dimer formation and the subsequent nuclear translocation and transcriptional activity (8). Similarly, Decker and Kovarik proposed that STAT3 phosphorylation on Ser727 either inhibits tyrosine phosphorylation or increases tyrosine dephosphorylation (9). A negative relationship between STAT3 serine and tyrosine phosphorylation has also been suggested by Venkatasubbarao et al (10) and Wakahara et al (63), who described that phospho-Ser727 determines the duration of STAT3 activity by enhancing dephosphorylation of phosphoTyr705 largely through TC45 phosphatase.

However, other investigators suggested that STAT3 serine phosphorylation is associated with increased nuclear translocation and serves to maximize transcriptional activity $(6,64)$. Hazan-Halevy et al reported that constitutive phosphorylation of STAT3 on Ser727 residues is a critical event in chronic lymphocytic leukemia (CLL) and may serve as a potent therapeutic target (64). Miyakoshi et al showed that FBS treatment of mouse hepatic carcinoma cells induced STAT3 phosphorylation on Ser727 via MAPK activation, followed by STAT3 nuclear translocation and cell proliferation (66). In contrast, IL- 6 treatment induced STAT3 phosphorylation on Tyr705 phosphorylation through JAK activation without increasing STAT3 nuclear translocation and cell proliferation. Sakaguchi et al investigated the oncogenic role of Ser727 STAT3 in melanoma cells and suggested that constitutive Ser 727 phosphorylation, partially mediated by the B-Raf-MEK-Erk1/2 pathway, has a role in the regulation of cell survival activity and nuclear translocation of STAT3 in melanocytes (65). Moreover, Gough et al proposed that the MEK-ERK pathway is required for activated Ras-induced phosphorylation of STAT3 on Ser727 and that mitochondrial STAT3 is one of the critical substrates of the Ras-MEK-ERKaxis during cellular transformation (35).
One of the major downstream signaling routes of MAPKs are STAT proteins and MAPKs have been implicated in the regulation of STAT proteins through crosstalk signaling (9).

Considering the significance of STAT3 and ERK signaling in several types of cancer, the possibility of a crosstalk between these two major oncogenic pathways in oral cancer was explored in the present study. In particular, changes in the expression and activation status of Erk1/2 MAPK and their effect on STAT3 tyrosine and/or serine phosphorylation and total STAT3 levels in OSCC cell lines were investigated. Chemical inhibition (via U0126) or selective targeting (via siRNA) of Erk1/2 MAPK downregulated STAT3 serine phosphorylation in both cell lines used; in addition, a moderate increase in STAT3 tyrosine (Y705) phosphorylation was observed in oral SCC9 cells. In contrast, induction of ERK in OSCC cells resulted in upregulation of STAT3 serine phosphorylation and downregulation of STAT3 tyrosine phosphorylation. Previous studies suggested that a crosstalk exists between ERK and STAT3, indicating that ERK either induces Ser727 phosphorylation $(8,28,35)$ or downregulates IL-6-mediated STAT3 signaling (29). In pancreatic cancer cells, ERK activation has been associated with STAT3 negative regulation manifested by decreased tyrosine phosphorylation levels (10). Similar results were obtained by Tkach et al, who found that U0126 suppresses phosphorylation of STAT3 on Ser727 in breast cancer cells (33). In addition, Wierenga et al, indicated that U0126 abrogated the EPO-mediated STAT3 Ser727 phosphorylation without an effect on tyrosine phosphorylation in erythroid cells (67). Nelson et al found that the combination of nifuroxazide and U0126 inhibits STAT3 tyrosine phosphorylation in multiple myeloma cells (68). Furthermore, Chen et al reported that Erk1/2 activation phosphorylates STAT3 on Ser727 and regulates cell proliferation in human bladder cancer cells (34). On the other hand, Sumimoto et al reported that U0126 inhibits ERK phosphorylation, but not STAT3 phosphorylation on Ser727 or Tyr705 residues in human melanoma cells (69). It can be hypothesized that the significance of Erk1/2 and STAT3 crosstalk differs according to the cancer cell types studied.

In summary, our data are supportive of the oncogenic potential of Erk1/2 in OSCC, which appears to contribute to cell proliferation. It is possible that pharmacologic inhibition of Erk1/2 activity or targeting Erk1/2 genes by gene therapy may offer an alternative strategy for the treatment of patients with OSCC. On the other hand, the oncogenic STAT3 constitutive signaling in OSCC cells appears to be negatively regulated by Erk1/2. The Erk1/2-STAT3 crosstalk appears to involve mainly ERK-induced upregulation of STAT3 Ser727 phosphorylation while Tyr705 phosphorylation does not exhibit major changes. It is possible that the role of Erk $1 / 2$ in STAT3 modulation varies according to the type and status of the cells studied, indicating the need to identify the role of MAPK activation in relation to STAT3 signaling in specific cell types.

\section{Acknowledgements}

This study was co-financed by the European Union (European Social Fund - ESF) and Greek national funds through the Operational Program 'Education and Lifelong Learning' of the 
National Strategic Reference Framework (NSRF) - Research Funding Program: Heracleitus II. Investing in knowledge society through the European Social Fund.

\section{References}

1. Curado MP and Hashibe M: Recent changes in the epidemiology of head and neck cancer. Curr Opin Oncol 21: 194-200, 2009.

2. Molinolo AA, Amornphimoltham P, Squarize $\mathrm{CH}$, et al: Dysregulated molecular networks in head and neck carcinogenesis. Oral Oncol 45: 324-334, 2009.

3. Choi S and Myers JN: Molecular pathogenesis of oral squamous cell carcinoma: implications for therapy. J Dent Res 87: 14-32, 2008.

4. Bromberg J and Darnell JE Jr: The role of STATs in transcriptional control and their impact on cellular function. Oncogene 19: 2468-2473, 2000.

5. Rane SG and Reddy EP: Janus kinases: components of multiple signaling pathways. Oncogene 19: 5662-5679, 2000.

6. Aggarwal BB, Kunnumakkara AB, Harikumar KB, et al: Signal transducer and activator of transcription-3, inflammation, and cancer: how intimate is the relationship? Ann NY Acad Sci 1171: 59-76, 2009

7. Reich NC and Liu L: Tracking STAT nuclear traffic. Nat Rev Immunol 6: 602-612, 2006.

8. Chung J, Uchida E, Grammer TC and Blenis J: STAT3 serine phosphorylation by ERK-dependent and -independent pathways negatively modulates its tyrosine phosphorylation. Mol Cell Biol 17: 6508-6516, 1997.

9. Decker T and Kovarik P: Serine phosphorylation of STATs Oncogene 19: 2628-2637, 2000.

10. Venkatasubbarao K, Choudary A and Freeman JW: Farnesyl transferase inhibitor (R115777)-induced inhibition of STAT3(Tyr705) phosphorylation in human pancreatic cancer cell lines require extracellular signal-regulated kinases. Cancer Res 65: 2861-2871, 2005.

11. Lim CP and Cao X: Serine phosphorylation and negative regulation of STAT3 by JNK. J Biol Chem 274: 31055-31061, 1999.

12. O'Shea JJ, Gadina M and Schreiber RD: Cytokine signaling in 2002: new surprises in the Jak/Stat pathway. Cell 109: S121-S131, 2002.

13. Bromberg J: Stat proteins and oncogenesis. J Clin Invest 109: $1139-1142,2002$

14. Song JI and Grandis JR: STAT signaling in head and neck cancer. Oncogene 19: 2489-2495, 2000.

15. Grandis JR, Drenning SD, Zeng Q, et al: Constitutive activation of Stat 3 signaling abrogates apoptosis in squamous cell carcinogenesis in vivo. Proc Natl Acad Sci USA 97: 4227-4232, 2000.

16. Nikitakis NG, Siavash H and Sauk JJ: Targeting the STAT pathway in head and neck cancer: recent advances and future prospects. Curr Cancer Drug Targets 4: 637-651, 2004.

17. Kijima T, Niwa H, Steinman RA, et al: STAT3 activation abrogates growth factor dependence and contributes to head and neck squamous cell carcinoma tumor growth in vivo. Cell Growth Differ 13: 355-362, 2002.

18. Leeman RJ, Lui VW and Grandis JR: STAT3 as a therapeutic target in head and neck cancer. Expert Opin Biol Ther 6: 231-241, 2006.

19. Siavash H, Nikitakis NG and Sauk JJ: Abrogation of IL-6mediated JAK signalling by the cyclopentenone prostaglandin $15 \mathrm{~d}-\mathrm{PGJ}(2)$ in oral squamous carcinoma cells. Br J Cancer 91: 1074-1080, 2004.

20. Lee TL, Yeh J, Van Waes C and Chen Z: Epigenetic modification of SOCS-1 differentially regulates STAT3 activation in response to interleukin-6 receptor and epidermal growth factor receptor signaling through JAK and/or MEK in head and neck squamous cell carcinomas. Mol Cancer Ther 5: 8-19, 2006.

21. Naher L, Kiyoshima T, Kobayashi I, et al: STAT3 signal transduction through interleukin-22 in oral squamous cell carcinoma. Int J Oncol 41: 1577-1586, 2012.

22. Jewett A, Head C and Cacalano NA: Emerging mechanisms of immunosuppression in oral cancers. J Dent Res 85: 1061-1073, 2006.

23. Lai SY and Johnson FM: Defining the role of the JAK-STAT pathway in head and neck and thoracic malignancies: implications for future therapeutic approaches. Drug Resist Updat 13: 67-78, 2010 .
24. Maggioni D, Gaini R, Nicolini G, et al: MAPKs activation in head and neck squamous cell carcinomas. Oncol Rev 5: 223-231, 2011.

25. Johnson GL and Lapadat R: Mitogen-activated protein kinase pathways mediated by ERK, JNK, and p38 protein kinases. Science 298: 1911-1912, 2002.

26. Pearce AK and Humphrey TC: Integrating stress-response and cell-cycle checkpoint pathways. Trends Cell Biol 11: 426-433, 2001.

27. Dunn C, Wiltshire C, MacLaren A and Gillespie DA: Molecular mechanism and biological functions of c-Jun N-terminal kinase signalling via the c-Jun transcription factor. Cell Signal 14: 585-593, 2002.

28. Jain N, Zhang T, Fong SL, et al: Repression of Stat3 activity by activation of mitogen-activated protein kinase (MAPK). Oncogene 17: 3157-3167, 1998.

29. Sengupta TK, Talbot ES, Scherle PA and Ivashkiv LB: Rapid inhibition of interleukin- 6 signaling and Stat 3 activation mediated by mitogen-activated protein kinases. Proc Natl Acad Sci USA 95: 11107-11112, 1998.

30. Quadros MR, Peruzzi F, Kari C and Rodeck U: Complex regulation of signal transducers and activators of transcription 3 activation in normal and malignant keratinocytes. Cancer Res 64: 3934-3939, 2004

31. Kovarik P, Stoiber D, Eyers PA, et al: Stress-induced phosphorylation of STAT1 at Ser727 requires p38 mitogen-activated protein kinase whereas IFN-gamma uses a different signaling pathway. Proc Natl Acad Sci USA 96: 13956-13961, 1999.

32. Ahmed ST, Mayer A, Ji JD and Ivashkiv LB: Inhibition of IL-6 signaling by a p38-dependent pathway occurs in the absence of new protein synthesis. J Leukoc Biol 72: 154-162, 2002.

33. Tkach M, Rosemblit C,Rivas MA, et al:p42/p44MAPK-mediated Stat3Ser727 phosphorylation is required for progestin-induced full activation of Stat 3 and breast cancer growth. Endocr Relat Cancer 20: 197-212, 2013.

34. Chen RJ, Ho YS, Guo HR and Wang YJ: Rapid activation of Stat 3 and ERK1/2 by nicotine modulates cell proliferation in human bladder cancer cells. Toxicol Sci 104: 283-293, 2008.

35. Gough D, Koetz L and Levy D: The MEK-ERK pathway is necessary for serine phosphorylation of mitochondrial STAT3 and Ras-mediated transformation. PLoS One 8: e83395, 2013.

36. Xue P, Zhao Y, Liu Y, Yuan Q, et al: A novel compound RY10-4 induces apoptosis and inhibits invasion via inhibiting STAT3 through ERK-, p38-dependent pathways in human lung adenocarcinoma A549 cells. Chem Biol Interact 209: 25-34, 2014

37. Lee MH, Huang Z, Kim DJ, et al: Direct targeting of MEK $1 / 2$ and RSK2 by silybin induces cell-cycle arrest and inhibits melanoma cell growth. Cancer Prev Res 6: 455-465, 2013.

38. Aguzzi A, Maggioni D, Nicolini G, et al: MAP kinase modulation in squamous cell carcinoma of the oral cavity. Anticancer Res 29: 303-308, 2009.

39. Dhillon AS, Hagan S, Rath O and Kolch W: MAP kinase signalling pathways in cancer. Oncogene 26: 3279-3290, 2007.

40. Chen Z, Gibson TB, Robinson F, et al: MAP kinases. Chem Rev 101: 2449-2476, 2001.

41. Lee SH, Lee JW, Soung YH, et al: Colorectal tumors frequently express phosphorylated mitogen-activated protein kinase. APMIS 112: 233-238, 2004.

42. Mendoza MC,ErEE and Blenis J: The Ras-ERK and PI3K-mTOR pathways: cross-talk and compensation. Trends Biochem Sci 36: 320-328, 2011.

43. Anjum R and Blenis J: The RSK family of kinases: emerging roles in cellular signalling. Nat Rev Mol Cell Biol 9: 747-758, 2008.

44. Bessard A, Frémin C, Ezan F, et al: RNAi-mediated ERK2 knockdown inhibits growth of tumor cells in vitro and in vivo. Oncogene 27: 5315-5325, 2008.

45. Obajimi O and Melera P: Suppression of ERK1/2 with siRNA restores drug sensitivity in DU145 cells selected for resistance to AG2034 (abstract 77). Cancer Res 70: (Suppl 1), 2010.

46. Smalley KS: A pivotal role for ERK in the oncogenic behaviour of malignant melanoma? Int J Cancer 104: 527-532, 2003.

47. Gao J, Zhao Y, Lv Y, et al: Mirk/Dyrk1B mediates G0/G1 to $\mathrm{S}$ phase cell cycle progression and cell survival involving MAPK/ ERK signaling in human cancer cells. Cancer Cell Int 13: 2, 2013.

48. Balmanno K and Cook SJ: Tumour cell survival signalling by the ERK1/2 pathway. Cell Death Differ 16: 368-377, 2009.

49. Lin YC, Wu MH, Wei TT, et al: Degradation of epidermal growth factor receptor mediates dasatinib-induced apoptosis in head and neck squamous cell carcinoma cells. Neoplasia 14: $463-475,2012$ 
50. Ji WT, Chen HR, Lin $\mathrm{CH}$, et al: Monocyte chemotactic protein 1 (MCP-1) modulates pro-survival signaling to promote progression of head and neck squamous cell carcinoma. PLoS One 9: e88952, 2014.

51. Wang L, Liu T, Nishioka M, et al: Activation of ERK1/2 and cyclin D1 expression in oral tongue squamous cell carcinomas: relationship between clinicopathological appearances and cell proliferation. Oral Oncol 42: 625-631, 2006.

52. Bancroft CC, Chen Z, Dong G, et al: Coexpression of proangiogenic factors IL-8 and VEGF by human head and neck squamous cell carcinoma involves coactivation by MEK-MAPK and IKK-NF-kappaB signal pathways. Clin Cancer Res 7: 435-442, 2001.

53. Duvvuri U, Shiwarski DJ, Xiao D, et al: TMEM16A induces MAPK and contributes directly to tumorigenesis and cancer progression. Cancer Res 72: 3270-3281, 2012.

54. Li B, Lu L, Zhong M, Tan XX, et al: Terbinafine inhibits KSR1 and suppresses Raf-MEK-ERK signaling in oral squamous cell carcinoma cells. Neoplasma 60: 406-412, 2013.

55. Lavoie JN, L'Allemain G, Brunet A, et al: Cyclin D1 expression is regulated positively by the $\mathrm{p} 42 / \mathrm{p} 44 \mathrm{MAPK}$ and negatively by the p38/HOGMAPK pathway. J Biol Chem 271: 20608-20616, 1996.

56. Zhao Y, Lv M, Lin H, et al: Rho-associated protein kinase isoforms stimulate proliferation of vascular smooth muscle cells through ERK and induction of cyclin D1 and PCNA. Biochem Biophys Res Commun 432: 488-493, 2013.

57. Si H, Peng C, Li J, Wang X, et al: RNAi-mediated knockdown of ERK1/2 inhibits cell proliferation and invasion and increases chemosensitivity to cisplatin in human osteosarcoma U2-OS cells in vitro. Int J Oncol 40: 1291-1297, 2012.

58. Dumesic PA, Scholl FA, Barragan DI and Khavari PA: Erk1/2 MAP kinases are required for epidermal $\mathrm{G} 2 / \mathrm{M}$ progression. J Cell Biol 185: 409-422, 2009.

59. Judd NP, Winkler AE, Murillo-Sauca O, et al: ERK1/2 regulation of CD44 modulates oral cancer aggressiveness. Cancer Res 72: 365-374, 2012.
60. Katada K, Tomonaga T, Satoh M, et al: Plectin promotes migration and invasion of cancer cells and is a novel prognostic marker for head and neck squamous cell carcinoma. J Proteomics 75: 1803-1815, 2012

61. Zuo JH, Zhu W, Li MY, et al: Activation of EGFR promotes squamous carcinoma SCC10A cell migration and invasion via inducing EMT-like phenotype change and MMP-9-mediated degradation of E-cadherin. J Cell Biochem 112: 2508-2517, 2011.

62. Li R, You S, Hu Z, et al: Inhibition of STAT3 by niclosamide synergizes with erlotinib against head and neck cancer. PLoS One 8: e74670, 2013.

63. Wakahara R, Kunimoto H, Tanino K, et al: Phospho-Ser727 of STAT3 regulates STAT3 activity by enhancing dephosphorylation of phospho-Tyr705 largely through TC45. Genes Cells 17: 132-145, 2012.

64. Hazan-Halevy I, Harris D, Liu Z, Liu J, et al: STAT3 is constitutively phosphorylated on serine 727 residues, binds DNA, and activates transcription in CLL cells. Blood 115: 2852-2863, 2010.

65. Sakaguchi M, Oka M, Iwasaki T, et al: Role and regulation of STAT3 phosphorylation at Ser727 in melanocytes and melanoma cells. J Invest Dermatol 132: 1877-1885, 2012.

66. Miyakoshi M, Yamamoto M, Tanaka $\mathrm{H}$ and Ogawa $\mathrm{K}$ : Serine 727 phosphorylation of STAT3: an early change in mouse hepatocarcinogenesis induced by neonatal treatment with diethylnitrosamine. Mol Carcinog 53: 67-76, 2014.

67. Wierenga AT, Vogelzang I, Eggen BJ and Vellenga E: Erythropoietin-induced serine 727 phosphorylation of STAT3 in erythroid cells is mediated by a MEK-, ERK-, and MSK1dependent pathway. Exp Hematol 31: 398-405, 2003.

68. Nelson EA, Walker SR, Kepich A, et al: Nifuroxazide inhibits survival of multiple myeloma cells by directly inhibiting STAT3. Blood 112: 5095-5102, 2008

69. Sumimoto H, Imabayashi $F$, Iwata $T$ and Kawakami Y: The BRAF-MAPK signaling pathway is essential for cancer-immune evasion in human melanoma cells. J Exp Med 203: 1651-1656, 2006. 